\section{Características de vizinhança e prática de atividade física: úma revisão sistemática}

\section{Neighborhood environment and physical activity: a systematic review}

Miguel Ataide Pinto da Costa

Maria de Jesus Mendes da Fonseca ${ }^{2}$

Sergio Henrique Almeida da Silva Júnior ${ }^{3}$

Ana Glória Godói Vasconcelos ${ }^{2}$

\section{RESUMO}

O objetivo do estudo foi investigar a relação entre características de vizinhança e a prática de atividade Física em adultos, por meio de revisão sistemática. Foram utilizadas as bases de dados Scopus, Web of Science e Pubmed, acessadas em fevereiro de 2013, com os seguintes descritores ("Residence Characteristics"[Mesh] OR Neighborhood [all field]) AND ("Sedentary Lifestyle"[Mesh] OR Physical activity [all fields] OR Motor activity [Mesh]) na base Pubmed e nos demais sintaxes correspondentes. Na busca inicial, foram encontrados 5779 (sendo 2257 duplicatas). Após análise e aplicação de critérios de inclusão 19 artigos fizeram parte da revisão. O principal instrumento utilizado para mensurar a atividade física foi o IPAQ (16 artigos). Em relação aos questionários para mensurar percepção de vizinhança foram identificados 5 instrumentos diferentes. A análise da associação entre atividade física e características de vizinhança apontou diversas inconsistências. Independentemente do instrumento utilizado, ao analisar apenas o constructo de cada característica de vizinhança, os resultados dos estudos foram divergentes ou inconsistentes, não indicando ainda uma sólida relação entre as características de vizinhança e a prática de atividade física. A diversidade de populações e metodologias para mensuração de características de vizinhança podem ser um dos motivos das diferenças apontadas. Este estudo aponta uma lacuna importante nos estudos de associação entre a prática de atividade física e características de vizinhança. $\mathrm{O}$ não uso de análises multinível nos estudos analisados pode ser apontado como um motivo para as associações não significativas ou inconsistências encontradas entre os estudos.

\section{PALAVRAS-CHAVE}

Características de vizinhança; Estilo de vida sedentário; Atividade motora.

\begin{abstract}
The aim of this study was to investigate the relationship between characteristics of neighborhood context and the practice of physical activity in adults through a systematic review. The Scopus, Web of Science and Pubmed database, were accessed in February 2013. We're used the syntax ("Residence Characteristics"[Mesh] OR Neighborhood [all field]) AND ("Sedentary Lifestyle"[Mesh] OR Physical activity [all fields] OR Motor activity [Mesh]) in Pubmed database and a correspondent syntax in the others. The initial search found 5779 (2257 being duplicates). After analysis and application of inclusion criteria 19 articles were included in the review. The main instrument used for measuring physical activity was the IPAQ (16 articles). Regarding questionnaires to measure perceived neighborbood, 5 different instruments were identified. The analysis of the association between physical activity and context characteristics pointed out several inconsistencies, regardless of the instrument used to analyze, not indicating a strong relationship between neighborhood characteristics and physical activitypractice. The diversity of populations and methodologies to measure neighborhood characteristics may be one of the reasons for the differences noted. This study bighlights an important gap in studies of association between physical activity and characteristics of the neighborbood context. No use of multilevel analyzes in the analyzed studies can be seen as a reason for no significant associations or inconsistencies found between studies.
\end{abstract}

\section{KEYWORDS}

Residence Characteristics; Sedentary Lifestyle; Motor activity.
Rev Bras Ativ Fís Saúde p. 113-129 DOI

http://dx.doi.org/10.12820/rbafs.v.20n2p113

1 Professor do Colégio Pedro II, departamento de Educação Física, Rio de Janeiro - RJ, Brasil.

2 FIOCRUZ - ENSP, departamento de métodos quantitativos, Rio de Janeiro - RJ, Brasil.

3 Professor do Instituto Benjamin Constant, Rio de Janeiro-RJ, Brasil. 


\section{INTRODUÇÃO}

Estudos de intervenção e observacionais sugerem que a prática de atividade física reduz substancialmente o risco de ocorrência de diversas doenças (tais como doenças coronarianas, acidentes vasculares cerebrais, diabetes, etc. $)^{1-2}$, participa também do processo de redução e controle de peso ${ }^{3}$, , diminui os sintomas de ansiedade, depressão, está associada com um menor número de hospitalizações, visitas médicas, uso de medicamentos ${ }^{4}$ e tem influência no consumo regular de frutas, legumes e verduras ${ }^{5}$. Apesar dos benefícios desta prática uma significativa parcela de individuos está se tornando cada vez mais inativos e não praticam níveis recomendados de atividade física ${ }^{6}$.

O processo de urbanização tem impactos positivos e negativos nas condições de vida (como maior acesso a bens de consumo e serviços, e crescimento desordenado) gerando diferenças sociais que repercutem na saúde. A partir do final da década de 80 e início da década de 90, observou-se um aumento dos estudos teóricos e empíricos que investigaram a relação entre fatores contextuais e desfechos em saúde pública seguindo o pressuposto de que o lugar em que se vive, para além das características individuais, contribui para melhorias nas condições de saúde ${ }^{7-9}$.

Existem evidências de que características ambientais podem influenciar a saúde e comportamentos relacionados à saúde, como a prática de vários tipos de atividade física ${ }^{10}$. Muitas formas de atividades físicas rotineiras são praticadas em lugares comuns como ruas, praças e calçadas de bairros, ou são realizadas preferencialmente em locais próximos as residências. Entretanto, estudos que têm investigado a relação entre a prática de atividade física e as características de vizinhança (como ruas com calçadas, adequado controle de tráfego, passagem para pedestres, segurança, iluminação pública, etc.) ou a percepção destas características por moradores de uma mesma região, encontram resultados ainda inconsistentes ao analisar esta relação ${ }^{11}$.

Observa-se nos últimos anos um aumento dos estudos de características do ambiente e sua influência nos processos de desenvolvimento de doenças assim como o sedentarismo e a inatividade física. Porém ainda não há estudos conclusivos que apontem associação clara entre a prática de atividade física e as diferentes características de vizinhança e nem como este processo ocorre, sendo assim necessárias e relevantes novas investigações.

O objetivo do presente estudo foi identificar e descrever questionários ou escalas auto-referidas utilizados para mensuração de características de vizinhança e investigar a relação entre estas características e a prática de atividade física em adultos, por meio de revisão sistemática, considerando estudos em que a prática de atividade física fosse a variável de desfecho. Espera-se, com esta revisão, descrever o estado atual das pesquisas epidemiológicas sobre esta associação, identificando lacunas que poderão ser preenchidas por pesquisas futuras na área. Em particular, buscou-se verificar quais são as principais características de vizinhança utilizadas nas pesquisas de associação com a prática de atividade física, a consistência e significância estatística das associações encontradas e discutir e comparar os resultados entre os diversos estudos. 


\section{MÉTODOS}

\section{Estratégia de Busca}

Investigou-se a associação entre caracteristicas de vizinhança e a prática de atividade física (como desfecho), conforme a metodologia proposta por Higgins \& Green ${ }^{12}$. Para este fim, foram utilizadas as bases de dados Scopus, Web of Science e Pubmed, acessadas em fevereiro de 2013. A estratégia de busca na base Pubmed foi baseada na seguinte chave: ("Residence Characteristics"[Mesh] OR Neighborhood [all field]) AND ("Sedentary Lifestyle"[Mesh] OR Physical activity [all fields] OR Motor activity [Mesh]). Para as demais bases de dados foram utilizadas as sintaxes correspondentes específicas de cada base de dados.

\section{Critérios de Inclusão/Exclusão}

Não houve limitação de data de publicação na revisão sistemática, todos os artigos encontrados (em qualquer data de publicação disponível nas bases de dados) poderiam ser incluídos na revisão. Também não houve restrição de língua em que o texto original poderia estar escrito.

Na primeira análise, foram verificados títulos e resumos dos artigos e para inclusão inicial na revisão foram identificados estudos que mencionaram tratarse de pesquisa que investigava a relação entre as características de vizinhança com a prática de atividade física, sedentarismo ou inatividade física, sendo incluídos apenas estudos com adultos (faixa etária de 18 anos ou mais).

Nesta primeira análise também foram excluídas as revisões sistemáticas, estudos em populações com doenças específicas (portadores de AIDS, câncer, amputados, etc.), pois estas impossibilitariam comparações relativas principalmente à atividade física, e estudos que não utilizassem a atividade física definida de forma global, mas apenas uma modalidade específica de atividade física (ciclismo, corrida, etc.). Porém, foram incluídos estudos que tratassem apenas da prática de caminhada por ser essa uma atividade física considerada de prática simples e um importante componente da prática de atividade física da população em geral (tanto para lazer como para transporte).

Os títulos e resumos dos artigos foram avaliados por dois revisores de forma independente, para verificar se atendiam aos critérios para inclusão na pesquisa, no caso de discordâncias os mesmos foram avaliados por um terceiro revisor.

Posteriormente, os artigos selecionados foram avaliados na íntegra e excluíram-se da revisão os estudos experimentais, estudos que não tinham a prática de atividade física como variável de desfecho, artigos que não apresentassem um modelo de análise ajustada por variáveis de confundimento e estudos que não aferiram a(s) variável(eis) de características de vizinhança e a prática de $\mathrm{AF}$ por meio de uma escala ou questionários validados.

\section{Avaliação dos estudos}

Os estudos foram analisados com o objetivo de identificar em qual sentido se orientavam as associações encontradas, buscando verificar diferenças e semelhanças nos resultados, diferenças relativas aos métodos e instrumentos de análise empregados, analisar e comparar os modelos de análises ajustadas, verificar quais variáveis eram utilizadas como fatores de confundimento e quais os métodos mais utilizados na definição das características de vizinhança e da prática de atividade física. 
As referências identificadas foram armazenadas e processadas por meio do programa JabRef@ 2.9.2.

\section{RESULTADOS}

$\mathrm{Na}$ busca inicial, feita apenas com os descritores, foram encontrados 5779 artigos (1756 na base scopus, 1835 em web of science e 2188 na base pubmed), e deste total, 2257 eram duplicatas. Na primeira análise, foram excluídos 3310 artigos que não atenderam aos critérios de inclusão, por meio da leitura dos títulos e resumos dos artigos, mantendo-se assim 212 artigos para a análise de texto integral.

$\mathrm{Na}$ segunda etapa, os 212 artigos restantes foram analisados e destes 193 foram excluídos por se tratarem de estudos de intervenção, por não utilizar escalas ou questionários para a construção da(s) variável(is) de características de vizinhança, não tratar da variável atividade física como desfecho ou não apresentar modelo de análise ajustada por fatores de confusão. Assim, 19 artigos foram selecionados e fizeram parte da revisão final (Figura 1).

Em relação à prática de atividade física o principal instrumento utilizado foi o International Physical Activity Questionnarie (IPAQ), tanto em seu formato longo como em seu formato curto, sendo utilizado em 16 artigos. Em cada um dos três artigos restantes foram utilizados instrumentos diferentes: (1) módulo corresponde a atividade física do inquérito denominado BRFSS (Behavioral Risk Factor Surveillance System); (2) SQUASH (Short Questionnaire to Assess Health-enhancing physical activity); (3) Physical Activity Scale for the Elderly (As Tabelas 1 e 2 mostram os resultados agrupados e separados por estudo, respectivamente).

Em relação aos questionários utilizados para mensurar as características de vizinhança foram identificados os seguintes instrumentos: Neighborhood Environment Walkability Scale (NEWS), International Physical Activity Questionnarie concebido para obter percepções de moradores de uma mesma vizinhança em relação à caracteristicas previamente determinadas pela literatura associadas a uma frequência maior de prática de caminhada e uso de bicicleta. O mesmo consiste em pouco mais de 60 itens separados em oito aspectos: tipos de residências na vizinhança; lojas, facilidades e comércio em geral; acesso a serviços; características da ruas; lugares para caminhar e pedalar ; características do entorno das vizinhanças; segurança no trânsito; segurança contra crimes; satisfação com a vizinhança. Muitos autores que utilizaram este intrumento em suas analises optaram por não utilizar as categorias previamente propostas pelos autores do instrumento ou não utilizaram todas as perguntas do questionário, o que dificulta a comparação entre os diversos estudos.

International Physical Activity Prevalence Study (2002). O questionário define como vizinhança os locais de acesso a pé num tempo máximo de $15 \mathrm{minu}$ tos de casa e consiste em 17 itens (16 em escala de Likert uma pergunta aberta) que englobam os seguintes aspectos: densidade residencial, acesso aos destinos, infra-estrutura do bairro, qualidades estéticas, ambiente social, conectividade de ruas, segurança da vizinhança e número de veículos por residência.

atividade física (a) Características da vizinhança: em seis itens, os entrevistados foram solicitados a caracterizar as pessoas em seu bairro com quatro opções de resposta variando de "nada fisicamente ativo" para "muito ativos fisicamen- 
5779 Estudos identificados nas bases de dados Pubmed / Web of Science / Scopus

Estudos duplicados ( $\mathrm{n}=2257$ )

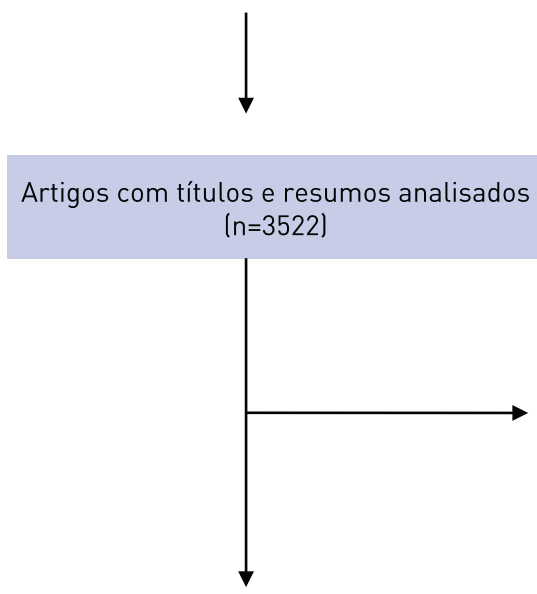

Artigos com análise de texto integral ( $n=212$ )

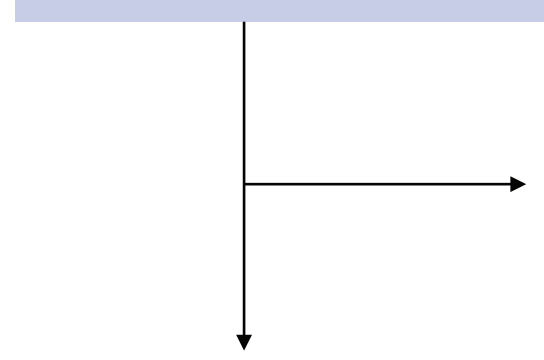

Artigos que entraram na revisão final $(n=19)$
Artigos excluídos por tratarem apenas de um dos dois objetos foco do estudo (AF ou contexto de vizinhançal, revisões sistemáticas, artigos que tratavam de crianças ou adolescentes e artigos que tratavam de populações com especificidades que impossibilitariam maiores

comparações com diferentes estudos lex: portadores de AIDS, câncer, distúrbios mentais, etc.). $(n=3310)$
Artigos excluídos por se tratarem de estudos de intervenção, por não utilizar escalas ou questionários para a

construção da variável AF como desfecho ou não apresentar modelo de análise ajustada por fatores de confusão. ( $n=193$ )

FIGURA 1 - Fluxograma da revisão de literatura.

te." Os entrevistados avaliaram seu bairro como um "bom lugar para caminhar" com quatro opções de resposta que vão desde "não agradável” a "muito agradável." Também era descrita a iluminação em seu bairro para andar à noite com cinco opções de resposta variando de "muito ruim" a "muito bom", além de segurança com relação a crimes com quatro opções de resposta variando de "nada segura" para "extremamente seguro", se a maioria das pessoas em sua vizinhança pode ser confiável ( $\operatorname{sim} /$ não), e se o seu bairro tem calçadas ( $\operatorname{sim} /$ não); (b) Instalações na vizinhança: Os entrevistados foram questionados sobre o uso de várias instalações de sua comunidade para a atividade física. Estas incluíram centros de recreação, escolas públicas que estão abertas para recreação, áreas de recreação privadas, trilhas para caminhada, parques, playgrounds ou campos esportivos e shopping centers. Os respondentes indicaram se eles usavam as instalações ( $\operatorname{sim}$ / não), ou se a comunidade não possuía esse tipo de instalação. 
TABELA 1 - Características bibliográficas agrupadas dos artigos analisados na revisão

\begin{tabular}{|c|c|c|}
\hline Características & $\mathbf{n}$ & $\%$ \\
\hline \multicolumn{3}{|l|}{ Ano de Publicação } \\
\hline 2005 & 1 & 5,3 \\
\hline 2008 & 1 & 5,3 \\
\hline 2009 & 6 & 31,6 \\
\hline 2010 & 1 & 5,3 \\
\hline 2011 & 3 & 15,8 \\
\hline 2012 & 6 & 31,6 \\
\hline 2013 & 1 & 5,3 \\
\hline \multicolumn{3}{|l|}{ Periódico de publicação } \\
\hline American Journal of Preventive Medicine & 2 & 10,5 \\
\hline Bmc Public Health & 1 & 5,3 \\
\hline European Journal of Public Health & 1 & 5,3 \\
\hline Health and Place & 1 & 5,3 \\
\hline International Journal of Behavioral Nutrition and Physical Activity & 3 & 15,8 \\
\hline Journal of Environmental and Public Health & 2 & 10,5 \\
\hline Preventing chronic disease & 1 & 5,3 \\
\hline Preventive Medicine & 5 & 26,3 \\
\hline Revista de Saúde Publica & 3 & 15,8 \\
\hline \multicolumn{3}{|l|}{ Países analisados nos estudos } \\
\hline Austrália* & 1 & 5,3 \\
\hline Bélgica* & 1 & 5,3 \\
\hline Alemanha & 2 & 10,5 \\
\hline Brasil* & 8 & 42,1 \\
\hline Canadá* & 1 & 5,3 \\
\hline China* & 1 & 5,3 \\
\hline Colômbia* & 1 & 5,3 \\
\hline EUA* & 4 & 21,1 \\
\hline Japão * & 4 & 21,1 \\
\hline Lituânia* & 1 & 5,3 \\
\hline Nigéria & 1 & 5,3 \\
\hline Noruega* & 1 & 5,3 \\
\hline Nova Zelândia* & 1 & 5,3 \\
\hline Portugal & 1 & 5,3 \\
\hline Suécia* & 2 & 10,5 \\
\hline \multicolumn{3}{|l|}{ Características } \\
\hline \multicolumn{3}{|l|}{ Instrumento de mensuração de atividade física } \\
\hline BRFSS questionnaire & 1 & 5,3 \\
\hline IPAQ & 16 & 84,2 \\
\hline Physical Activity Scale for the Elderly & 1 & 5,3 \\
\hline SQUASH questionnaire & 1 & 5,3 \\
\hline \multicolumn{3}{|l|}{ Instrumento de mensuração de características de vizinhança } \\
\hline BRFSS questionnaire & 1 & 5,3 \\
\hline Instrumento próprio & 2 & 10,5 \\
\hline IPAQ - E & 8 & 42,1 \\
\hline NEWS & 8 & 42,1 \\
\hline \multicolumn{3}{|l|}{ Amostra } \\
\hline$<500$ & 5 & 26,3 \\
\hline $500-1500$ & 6 & 31,6 \\
\hline $1501-6000$ & 4 & 21,1 \\
\hline$>6000$ & 4 & 21,1 \\
\hline
\end{tabular}

*Países participantes de estudos com mais de um país analisado. 
TABELA 2 - Artigos selecionados para revisão sistemática com autores, periódico, local do estudo, ano de publicação e metodologia utilizada para caracterização de prática de atividade física (AF) e medida de vizinhança.

\begin{tabular}{|c|c|c|c|c|c|c|}
\hline Autores & Ano & Título & Periódico & $\begin{array}{l}\text { Local do } \\
\text { estudo }\end{array}$ & $\begin{array}{l}\text { Medidas } \\
\text { vizinhança }\end{array}$ & $\begin{array}{l}\text { Medida } \\
\text { de atividade física }\end{array}$ \\
\hline $\begin{array}{l}\text { Van Dyck } \\
\text { et al. }\end{array}$ & 2013 & $\begin{array}{l}\text { Perceived neighborhood environmental attributes } \\
\text { associated with adults' leisure-time physical activity: } \\
\text { Findings from Belgium, Australia and the USA }\end{array}$ & Health andPlace & $\begin{array}{l}\text { EUA, Austrália } \\
\text { Bélgica }\end{array}$ & NEWS & IPAQ \\
\hline Rech et al. & 2012 & $\begin{array}{l}\text { Neighborhood safety and physical inactivity in } \\
\text { adults from Curitiba, Brazil }\end{array}$ & $\begin{array}{l}\text { International } \\
\text { Journal of } \\
\text { Behavioral } \\
\text { Nutrition and } \\
\text { Physical Activity }\end{array}$ & $\begin{array}{l}\text { Brasil } \\
\text { (Curitiba) }\end{array}$ & NEWS & IPAQ \\
\hline $\begin{array}{l}\text { Corseuil } \\
\text { et al. }\end{array}$ & 2012 & $\begin{array}{l}\text { Safety from crime and physical activity among } \\
\text { older adults: A population-based study in Brazil }\end{array}$ & $\begin{array}{l}\text { Journal of } \\
\text { Environmental and } \\
\text { Public Health }\end{array}$ & $\begin{array}{l}\text { Brasil } \\
\text { (Florianópolis) }\end{array}$ & NEWS & IPAQ \\
\hline Giehl et al. & 2012 & $\begin{array}{l}\text { Physical activity and environment perception } \\
\text { among older adults: a population study in } \\
\text { Florianopolis, Brazil }\end{array}$ & $\begin{array}{l}\text { Revista De Saude } \\
\text { Publica }\end{array}$ & $\begin{array}{l}\text { Brasil } \\
\text { (Florianópolis) }\end{array}$ & NEWS & IPAQ \\
\hline $\begin{array}{l}\text { Oyeyemi } \\
\text { et al. }\end{array}$ & 2012 & $\begin{array}{l}\text { Perceived crime and traffic safety is related to } \\
\text { physical activity among adults in Nigeria }\end{array}$ & BmcPublic Health & $\begin{array}{l}\text { Nigéria } \\
\text { (Maiduguri) }\end{array}$ & IPAQ-E & $\begin{array}{l}\text { Acelerômetro } \\
\text { IPAQ (longo) }\end{array}$ \\
\hline $\begin{array}{l}\text { Tsunoda } \\
\text { et al. }\end{array}$ & 2012 & $\begin{array}{l}\text { Associations of physical activity with neighborhood } \\
\text { environments and transportation modes in older } \\
\text { Japanese adults }\end{array}$ & Preventive Medicine & $\begin{array}{l}\text { Japão } \\
\text { (Kasama) }\end{array}$ & IPAQ - E & $\begin{array}{l}\text { Physical } \\
\text { Activity Scale } \\
\text { for the Elderly }\end{array}$ \\
\hline $\begin{array}{l}\text { Wallmann } \\
\text { et al. }\end{array}$ & 2012 & $\begin{array}{l}\text { The association between physical activity and } \\
\text { perceived environment in German adults }\end{array}$ & $\begin{array}{l}\text { European Journal } \\
\text { of Public Health }\end{array}$ & Alemanha & IPAQ - E & IPAQ \\
\hline Parra et al. & 2011 & $\begin{array}{l}\text { Perceived environmental correlates of physical } \\
\text { activity for leisure and transportation in Curitiba, } \\
\text { Brazil }\end{array}$ & Preventive Medicine & $\begin{array}{l}\text { Brasil } \\
\text { (Curitiba) }\end{array}$ & NEWS & IPAQ (longo) \\
\hline $\begin{array}{l}\text { Florindo } \\
\text { et al. }\end{array}$ & 2011 & $\begin{array}{l}\text { Perception of the environment and practice of } \\
\text { physical activity by adults in a low socioeconomic } \\
\text { area }\end{array}$ & $\begin{array}{l}\text { Revista De Saude } \\
\text { Publica }\end{array}$ & $\begin{array}{l}\text { Brasil } \\
\text { (Ermelino } \\
\text { Matarazzo) }\end{array}$ & NEWS & IPAQ \\
\hline $\begin{array}{l}\text { Beenackers } \\
\text { et al. }\end{array}$ & 2011 & $\begin{array}{l}\text { Sports participation, perceived neighborhood } \\
\text { safety, and individual cognitions: how do they } \\
\text { interact? }\end{array}$ & $\begin{array}{l}\text { International } \\
\text { Journal of } \\
\text { Behavioral } \\
\text { Nutrition and } \\
\text { Physical Activity }\end{array}$ & $\begin{array}{l}\text { Alemanha } \\
\text { (Eindhoven) }\end{array}$ & $\begin{array}{l}\text { Instrumento } \\
\text { próprio }\end{array}$ & $\begin{array}{l}\text { SQUASH } \\
\text { questionnaire }\end{array}$ \\
\hline $\begin{array}{l}\text { Amorim } \\
\text { et al. }\end{array}$ & 2010 & $\begin{array}{l}\text { Physical Activity Levels According to Physical } \\
\text { and Social Environmental Factors in a Sample of } \\
\text { Adults Living in South Brazil }\end{array}$ & $\begin{array}{l}\text { Journal of Physical } \\
\text { Activity } \backslash \& \text { Health }\end{array}$ & $\begin{array}{l}\text { Brasil } \\
\text { (Pelotas) }\end{array}$ & NEWS & IPAQ \\
\hline $\begin{array}{l}\text { Kamada } \\
\text { et al. }\end{array}$ & 2009 & $\begin{array}{l}\text { Environmental correlates of physical activity in } \\
\text { driving and non-driving rural Japanese women }\end{array}$ & Preventive Medicine & Japao (Unnan) & IPAQ-E & IPAQ \\
\hline $\begin{array}{l}\text { Salvador } \\
\text { et al. }\end{array}$ & 2009 & $\begin{array}{l}\text { Perception of the environment and leisure-time } \\
\text { physical activity in the elderly }\end{array}$ & $\begin{array}{l}\text { Revista De Saúde } \\
\text { Publica }\end{array}$ & $\begin{array}{l}\text { Brasil } \\
\text { (Ermelino } \\
\text { Matarazzo) }\end{array}$ & NEWS & IPAQ \\
\hline Sallis et al. & 2009 & $\begin{array}{l}\text { Neighborhood Environments and Physical Activity } \\
\text { Among Adults in } 11 \text { Countries }\end{array}$ & $\begin{array}{l}\text { American Journal } \\
\text { of Preventive } \\
\text { Medicine }\end{array}$ & Multicêntrico* & IPAQ-E & IPAQ \\
\hline Inoue et al. & 2009 & $\begin{array}{l}\text { Association of physical activity and neighborhood } \\
\text { environment among Japanese adults }\end{array}$ & Preventive Medicine & $\begin{array}{l}\text { Japão } \\
\text { (Tokyo eHimeji) }\end{array}$ & IPAQ - E & IPAQ \\
\hline $\begin{array}{l}\text { Bergman } \\
\text { et al. }\end{array}$ & 2009 & $\begin{array}{l}\text { The association between health enhancing } \\
\text { physical activity and neighbourhood environment } \\
\text { among Swedish adults - a population-based } \\
\text { cross-sectional study }\end{array}$ & $\begin{array}{l}\text { International } \\
\text { Journal of } \\
\text { Behavioral } \\
\text { Nutrition and } \\
\text { Physical Activity }\end{array}$ & Suécia & IPAQ - E & IPAQ \\
\hline $\begin{array}{l}\text { Velasquez } \\
\text { et al. }\end{array}$ & 2009 & $\begin{array}{l}\text { Relationship of perceived environmental } \\
\text { characteristics to leisure-time physical activity } \\
\text { and meeting recommendations for physical } \\
\text { activity in Texas }\end{array}$ & $\begin{array}{l}\text { Preventing chronic } \\
\text { disease }\end{array}$ & $\begin{array}{l}\text { EUA } \\
\text { (Texas) }\end{array}$ & $\begin{array}{l}\text { BRFSS } \\
\text { questionnaire }\end{array}$ & $\begin{array}{l}\text { BRFSS } \\
\text { questionnaire }\end{array}$ \\
\hline $\begin{array}{l}\text { Santos et } \\
\text { al. }\end{array}$ & 2008 & $\begin{array}{l}\text { Physical activity and perceived environmental } \\
\text { attributes in a sample of Portuguese adults: } \\
\text { Results from the Azorean Physical Activity and } \\
\text { Health Study }\end{array}$ & Preventive Medicine & $\begin{array}{l}\text { Portugal } \\
\text { (Arquipélago } \\
\text { de Azorean) }\end{array}$ & IPAQ-E & IPAQ \\
\hline $\begin{array}{l}\text { Hoehner } \\
\text { et al. }\end{array}$ & 2005 & $\begin{array}{l}\text { Perceived and objective environmental measures } \\
\text { and physical activity among urban adults }\end{array}$ & $\begin{array}{l}\text { American Journal } \\
\text { of Preventive } \\
\text { Medicine }\end{array}$ & $\begin{array}{l}\text { EUA(St. Louis } \\
\text { e Savannah) }\end{array}$ & $\begin{array}{l}\text { Instrumento } \\
\text { próprio }\end{array}$ & IPAQ \\
\hline
\end{tabular}

*Bélgica, Brasil, Canadá, Colômbia, China (Hong Kong), Japão, Lituânia, Noruega,Nova Zelândia, Suécia e EUA 
Devido à diversidade de instrumentos e classificações para mensurar características de vizinhança, identificou-se a necessidade de realizar a análise das associações agrupando artigos que utilizaram metodologias semelhantes. Três artigos que usaram o IPAQ-E $\mathrm{E}^{13-15}$ definiram a mesma categorização, originalmente proposta pelos autores para classificação de características de vizinhança e IPAQ para mensuração de atividade física. Porém não houve nenhuma característica de vizinhança com associação significativa comum aos três artigos em nenhum dos desfechos de prática de atividade física analisados, prática de caminhada e prática total de atividade física (Tabela 3 descreve as OR's encontradas pelos estudos que utilizaram IPAQ-E).

Kamada e colaboradores (2009) ${ }^{13}$, nas análises ajustadas para identificar a associação entre características de vizinhança e a prática global de atividade física, verificaram que o conjunto de características de melhor "acesso a transporte público" foi associado significativamente a ser "suficientemente ativo" $(\mathrm{OR}=1.57$, IC95\% 1.01-2.44) assim como maior "presença de ciclovias" (OR=2.5, IC95\% 1.30-3.22) e melhor "qualidade estética da vizinhança" (OR=1.69, IC95\% 1.092.63). Tsunoda e colaboradores $(2012)^{14}$ verificaram que, para o mesmo desfecho, foi encontrada associação significativa nas categorias de características de vizinhança de melhor "acesso as instalações recreativas" e maior "presença de calçadas" (OR=1.67, IC95\% 1.07-2.62 e OR=1.76, IC95\% 1.07-2.89, respectivamente). Nas análises de Inoue e colaboradores (2009), a atividade física global mostrou-se associada com as categorias de melhor "acesso a comércio" (OR=2,32, IC95\% 1.47-3.68) e maior "presença de ciclovias" (OR=1,57, IC95\% 1.04-2.36), sendo esta a única associação comum entre os estudos.

Em relação ao desfecho "caminhada", analisado em Tsunoda e colaboradores $(2012)^{14}$ e Inoue e colaboradores (2009) ${ }^{15}$, não houve nenhum fator de associação significativo comum nas análises ajustadas. No primeiro estudo, a categoria de vizinhança "qualidades estéticas da vizinhança" apresentou associação direta significativa com "caminhar mais que $150 \mathrm{~min} / \mathrm{sem}$ ” (OR=2,00, IC95\% 1.33-3.02) e no segundo estudo foram encontradas associações significativas para as categorias maior "acesso a comercio" (OR=1,65, IC95\% $1.05-2.58)$ e maior "presença de calcadas" (OR=1,65, IC95\% 1.13-2.42) com o mesmo desfecho.

Destaca-se que em nenhum dos três estudos foi encontrada associação significativa de atividade física com as características de vizinhança relativas à "segurança relacionada a crimes" e a "segurança relacionada ao tráfego", ambas categorias teoricamente importantes nesta relação. Estes resultados foram destacados pelos respectivos autores em seus artigos.

Em contrapartida, Oyeyemi e colaboradores $(2012)^{16}$, que também utilizaram IPAQ-E (porém com adaptações) e IPAQ como instrumentos de mensuração, analisaram somente questões relacionadas à segurança das vizinhanças e verificaram que nas análises ajustadas "segurança relacionada a crimes" esteve fortemente e diretamente associada com a prática de caminhada $(\mathrm{OR}=5.92$, IC95\% 1.38-60.5 para percepção durante o dia e OR=7.00, IC95\% 2.71-18.0 para percepção durante a noite), porém os autores não encontraram associações significativas entre atividade física e "segurança relacionada ao tráfego".

Walman e colaboradores $(2012)^{17}$ apresentaram resultados de associação individualmente por cada pergunta do questionário. Assim verificou-se que para indivíduos moradores de áreas rurais, "dificuldades percebidas no trá- 
TABELA 3 - Artigos selecionados para revisão sistemática que utilizaram IPAQ-E para análise de características de vizinhança segundo as dimensões do questionário, OR de associação ajustada (comparando melhor percepção com percepções ruins como referência) com desfecho de prática de AF e IC95\%.

\begin{tabular}{|c|c|c|c|c|c|}
\hline & \multirow{2}{*}{$\begin{array}{c}\text { Kamada et al. (2009) } \\
\text { Atividade física } \\
\text { Global }\end{array}$} & \multicolumn{2}{|c|}{ Tsunoda et al. $(2012)^{\mathrm{b}}$} & \multicolumn{2}{|c|}{ Inoue et al. (2009) c } \\
\hline & & $\begin{array}{l}\text { Atividade física } \\
\text { Global }\end{array}$ & Caminhada & $\begin{array}{l}\text { Atividade física } \\
\text { Global }\end{array}$ & Caminhada \\
\hline Variáveis do IPAQ-E & OR (IC95\%) & OR (IC95\%) & OR (IC95\%) & OR (IC95\%) & OR (IC95\%) \\
\hline Segurança relativa a crimes & $1.10(0.70-1.72)$ & $0.99(0.61-1.62)$ & $0.94(0.60-1.48)$ & $1.37(0.92-2.04)$ & $1.30(0.87-1.94)$ \\
\hline Segurança relativa a tráfego & $0.93(0.59-1.47)$ & $1.29(0.81-2.05)$ & $1.46(0.96-2.21)$ & $1.01(0.69-1.48)$ & $0.80(0.55-1.17)$ \\
\hline Densidade residencial & $0.81(0.33-1.96)$ & $1.26(0.56-2.81)$ & $0.82(0.39-1.72)$ & $1.07(0.69-1.68)$ & $1.82(1.16-2.84)^{*}$ \\
\hline Acesso ao comércio & $1.14(0.72-1.80)$ & $1.14(0.73-1.79)$ & $0.67(0.45-0.98)$ & $2.32(1.47-3.68)^{*}$ & $1.65(1.05-2.58)^{*}$ \\
\hline Acesso a transporte público & $1.57(1.01-2.44)^{*}$ & $1.19(0.75-1.88)$ & $0.64(0.42-0.98)$ & $1.50(0.87-2.59)$ & $1.43(0.82-2.48)$ \\
\hline Acesso a locais para prática de AF & $1.17(0.75-1.81)$ & $1.67(1.07-2.62)^{*}$ & $1.20(0.80-1.80)$ & $1.09(0.75-1.58)$ & $1.14(0.79-1.66)$ \\
\hline Presença de calçadas & $1.35(0.87-2.10)$ & $1.76(1.07-2.89)^{*}$ & $0.73(0.48-1.13)$ & $1.39(0.95-2.04)$ & $1.65(1.13-2.42)^{*}$ \\
\hline Presença de ciclovias & $2.05(1.30-3.22)^{*}$ & $1.39(0.88-2.17)$ & $0.95(0.63-1.43)$ & $1.57(1.04-2.36)^{*}$ & $0.93(0.62-1.40)$ \\
\hline Estética de vizinhança & $1.69(1.09-2.63)^{*}$ & $2.13(1.35-3.35)^{*}$ & $2.00(1.33-3.02)^{*}$ & $1.38(0.94-2.02)$ & $1.04(0.71-1.50)$ \\
\hline Prática de AF pelos vizinhos & $1.16(0.75-1.80)$ & $1.83(0.98-3.43)$ & $1.04(0.62-1.76)$ & $1.35(0.92-1.99)$ & $1.05(0.72-1.55)$ \\
\hline \multirow[t]{3}{*}{ Quantidade de veículos por residência } & $0.82(0.52-1.31)$ & $1.49(0.57-3.87)$ & $0.72(0.33-1.61)$ & $1.47(0.93-2.32)$ & $1.54(0.99-2.41)$ \\
\hline & \multicolumn{2}{|c|}{ Oyeyemi et al. (2012)d } & \multicolumn{2}{|c|}{ Santos et al. (2008)e } & Sallis et al. (2009)f \\
\hline & \multicolumn{2}{|c|}{ Caminhada } & \multicolumn{2}{|c|}{ Atividade física Global } & $\begin{array}{l}\text { Atividade física } \\
\text { Global }\end{array}$ \\
\hline Variáveis do IPAQ-E & \multicolumn{2}{|c|}{ OR (IC95\%) } & \multicolumn{2}{|c|}{ OR (IC95\%) } & OR (IC95\%) \\
\hline Segurança relativa a crimes & \multicolumn{2}{|c|}{$5.92(1.38-60.59)^{*}$} & \multirow{2}{*}{\multicolumn{2}{|c|}{$\begin{array}{l}\text { Homens } 0.99(0.83-1.19) \\
\text { Mulheres } 1.01(0.85-1.21)\end{array}$}} & $\begin{array}{c}\text { Não } \\
\text { informado }\end{array}$ \\
\hline Segurança relativa a tráfego & Noturna & $(2.71-18.04)^{*}$ & & & $1.32(1.16-1.54)^{*}$ \\
\hline Densidade residencial & \multicolumn{2}{|c|}{$1.19(0.19-7.12)$} & & & Não informado \\
\hline Acesso ao comércio & \multicolumn{2}{|l|}{-} & & & $1.29(1.15-1.44)^{*}$ \\
\hline Acesso a transporte público & \multicolumn{2}{|l|}{-} & & & - \\
\hline Acesso a locais para prática de $\mathrm{AF}$ & \multicolumn{2}{|l|}{ 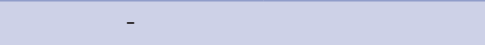 } & \multirow{2}{*}{\multicolumn{2}{|c|}{$\begin{array}{c}\text { Homens } 1.03 \\
(0.87-1.23) \\
\text { Mulheres 1.31 } \\
(1.12-1.55)^{*}\end{array}$}} & $1.16(1.05-1.27)^{*}$ \\
\hline Presença de calçadas & - & & & & $1.47(1.32-1.65)^{*}$ \\
\hline Presença de ciclovias & \multicolumn{2}{|l|}{ 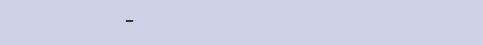 } & & & $1.21(1.10-1.33)^{*}$ \\
\hline Estética de vizinhança & \multicolumn{2}{|l|}{ 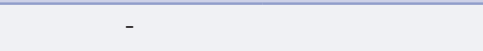 } & & & - \\
\hline Prática de AF pelos vizinhos & \multicolumn{2}{|l|}{-} & & & - \\
\hline
\end{tabular}

- Itens não avaliados, * p-valor < 0.05, a- Ajustado por idade, IMC, auto-avaliação de saúde e renda. b- Ajustado por idade, gênero, nível educacional, situação ocupacional ehistórico de doenças.

c- Ajustado por idade,gênero, situação ocupacional, nível educacional. d- Ajustado por idade, localização da vizinhança, nível educacional, situação ocupacional. e-Ajustado por idade, localização da vizinhança, nível educacional. f- Ajustado por idade, gênero, país de moradia.

fego" são empecilhos para prática de caminhada. Com relação à atividade física global, "boa conservação de ciclovia" foi associada a níveis mais altos de atividade física em área rural e "dificuldades percebidas no tráfego" foram associados tanto em área rural quanto em áreas urbanas. Nenhuma outra pergunta do questionário mostrou-se associada significativamente aos desfechos de prática de atividade física analisados.

Santos e colaboradores (2008) $)^{18}$, utilizando PCA (Principal Component Analysis) optaram por realizar uma agregação das características previamente estipuladas pelo questionário. A única categoria agregada que se mostrou 
associada (positivamente) à atividade física foi a que uniu "infra-estrutura", "acesso a destinos", "ambiente social" e "estética da vizinhança", porém essa associação só foi encontrada em mulheres (OR=1.31, IC95\% 1.12-1.55).

Bergman e colaboradores $(2009)^{19}$, também utilizando PCA, criaram novas categorizações próprias a partir das perguntas originais do IPAQ-E. Para o desfecho atividade física global, nenhuma associação foi encontrada com qualquer característica de vizinhança. Para o desfecho "caminhada", a categoria "oportunidades e estética" foi associada positivamente com "caminhar de 80 a $300 \mathrm{~min} / \mathrm{sem}$ " e "mais que $300 \mathrm{~min} / \mathrm{sem}$ ", e a categoria "medo de crimes" foi associada inversamente com "caminhar entre 80 a $300 \mathrm{~min} / \mathrm{sem}$ " (quanto menor o medo de crimes, maior a chance de praticar caminhada).

Sallis e colaboradores (2009) ${ }^{20}$ identificaram que a atividade física global foi significativamente relacionada a cinco das sete variáveis analisadas (OR comparando percepções positivas tendo como base de comparação percepções negativas): "quantidades de estabelecimento comerciais nas proximidades" (OR=1.29, IC95\% 1.15-1.44); "trânsito" (OR=1.32, IC95\% 1.16-1.54); "presença de calçadas" (OR=1.47, IC95\% 1.32-1.65); "facilidades para o uso de bicicletas" (OR=1.21, IC95\% 1.10-1.33); "acesso a estabelecimentos recreativos de baixo custo" (OR=1.16, IC95\% 1.05-1.27). As variáveis que não tiveram a associação confirmada foram "percepção de criminalidade" e "densidade residencial".

Dos artigos que utilizaram NEWS, como medida de características de vizinhança, destaca-se o uso do instrumento em população brasileira em sete dos oito artigos que participaram da revisão. Considerando todos os estudos que utilizaram este instrumento, verificou-se uma grande diversidade de categorizações e formas de apresentação dos resultados diferenciadas da proposta original dos autores do questionário. Assim, os estudos utilizando NEWS foram por demais heterogêneos, impossibilitando sua comparação através de tabelas descritivas.

Dois dos artigos que utilizaram NEWS analisaram somente questões relativas à percepção de segurança na vizinhança associada à prática de atividade física. Corseuil e colaboradores $(2012)^{10}$ verificaram nas análises ajustadas que maior "percepção de segurança para caminhar durante o dia" foi associada com altos níveis de atividade física de lazer ( $\mathrm{RP}=1.25$, IC95\% 1.02-1.53), assim como um escore proposto pelos autores de maior "segurança em geral" $(\mathrm{RP}=1.25$, IC95\% 1.01-1.54). Os autores também analisaram o desfecho "atividade física de transporte" e encontraram associação significativa com melhor percepção de "boa iluminação das ruas à noite" (RP=1.89, IC95\% 1.282.80). Rech e colaboradores $(2012)^{21}$, também analisando "atividade física de transporte" encontraram associação significativa entre esse desfecho e melhor "percepção de segurança para caminhar durante a noite" (RP=0.73, IC95\% 0.57-0.94, comparando "inseguro" com "seguro" - categoria de referência). Nenhuma outra associação foi encontrada nos dois artigos em relação aos demais desfechos de atividade física analisados ("caminhada de lazer", "atividade física global" e "atividade física de transporte").

Parra e colaboradores $(2011)^{22}$, também analisando características de vizinhança utilizando NEWS em seu formato original, verificaram nos modelos ajustados que alta percepção de "acessibilidade" (OR=1.40, IC95\% 1.00-1.80) e "estética da vizinhança" foram associados significativamente com "atividade 
física global". Níveis moderados de "percepção de segurança" também foram associados à "atividade física global" (OR=1.30, IC95\% 1.00-1.70), em contrapartida altos níveis de "segurança pessoal" não foram associados, porém esse resultado pode ser explicado pela pouca quantidade de indivíduos nessa categoria.

Os quatro artigos restantes que analisaram população brasileira utilizaram versões adaptadas da NEWS ou recategorizações próprias, o que dificulta maiores comparações entre os estudos. Destaca-se em Amorim e colaboradores $(2010)^{23}$ que dentre as 28 variáveis de vizinhança analisadas, apenas quatro mostraram-se associadas ao desfecho "não realizar caminhada em mais de $150 \mathrm{~min} / \mathrm{sem}$ " nos modelos ajustados, sendo estas "acúmulo de lixo na rua" ( $\mathrm{RP}=0.86$, IC95\% 0.75-0.97), "dificuldades com tráfego" (RP=1.18, IC95\% 1.04-1.33), "existência de área verde" ( $\mathrm{RP}=0.87$, IC95\% 0.81-0.94) e "existência de crime" ( $\mathrm{RP}=1.10$, IC95\% 1.00-1.22).

Em Florindo e colaboradores $(2011)^{24}$, apenas uma das variáveis analisadas foi associada com "atividade física de transporte", sendo esta um escore próprio de "sensação de segurança" em quatro níveis. A associação significativa foi encontrada apenas em indivíduos classificados no escore mais alto, comparados com indivíduos no escore mais baixo ( $R R=1.19$, IC95\% 1.07-1.33). Salvador e colaboradores (2009) $)^{25}$, analisando apenas idosos da mesma população, também utilizaram categorizações próprias para caracterização da vizinhança e verificaram que "boa percepção de segurança durante o dia" $(\mathrm{OR}=4.21$, IC95\% 1.29-13.8), "presença de quadras esportivas" (OR=2.95, IC95\% 1.237.09) e "convite de amigos para prática de atividade física" (OR=3.13, IC95\% 1.26-7.74) foram associados positivamente à "atividade física de lazer".

Giehl e colaboradores $(2012)^{26}$ verificaram que das 18 variáveis de características de vizinhança analisadas apenas três foram associadas significativamente à "atividade física de lazer" nos modelos ajustados. Foram estas a melhor percepção de "convite de amigos para prática de atividade física" ( $\mathrm{RP}=1.26$, IC95\% 1.03-1.43), "existência de ciclovias, vias, trilhas para pedestres de fácil acesso" (RP=1.25, IC95\% 1.03-1.43) e "clima limitador para prática de atividade física" ( $\mathrm{RP}=1.26, \mathrm{IC} 95 \%$ 1.05-1.52), resultado este que foi no sentido inverso do esperado.

Van Dick e colaboradores (2013) ${ }^{27}$ analisaram dados de três países em conjunto (EUA, Bélgica e Austrália) e verificaram que das sete características de vizinhança (originais do NEWS) apenas melhor percepção de "densidade residencial" foi associada significativamente com "atividade física de lazer".

Velasquez e colaboradores $(2009)^{28}$ foi o único artigo encontrado que fez análise utilizando o BRFSS questionnaire. Verificou-se que das 11 características de vizinhança analisadas nenhuma foi associada significativamente a prática de atividade física em homens. Para as mulheres, seis características analisadas mostraram associação significativa com pelo menos um dos desfechos de atividade física analisados (atingir níveis recomendados de atividade física ou praticar alguma atividade física), sendo estas melhor percepção de "prática de atividade física na vizinhança", "qualidade da vizinhança para prática de caminhada", "segurança relativa à crimes", "uso de locais públicos na vizinhança para prática de atividade física", "uso de locais privados na vizinhança para prática de atividade física" e "uso de escolas que são abertas para atividades recreativas".

Dois artigos utilizaram questionários próprios validados no âmbito do estudo para verificar a associação de atividade física e características de vi- 
zinhança. Beenackers e colaboradores $(2011)^{29}$, verificando a relação entre segurança na vizinhança e atividade física, encontraram que indivíduos que perceberam seu bairro como seguro eram duas vezes mais propensos a participar de práticas esportivas $(\mathrm{OR}=0.57$, IC95\% 0.42-0.77). Hoehner e colaboradores $(2005)^{30}$ encontraram em seu estudo que o fator mais associado à "atividade física de transporte" era ter destinos (trabalho, comércio, etc.) a uma distância suficiente pra ir caminhando (perguntada de forma subjetiva). Os autores destacam que nenhuma associação foi identificada entre "atividade física de lazer" e "presença de instalações recreativas", "segurança no trânsito", "estética da vizinhança", "segurança em relação à crimes", "existência de calçadas", "existências de parques ou áreas verdes" e "existência de ciclovias".

A Tabela 4 mostra os resultados de quinze estudos de forma agrupada, considerando as características de vizinhança mais citadas pelos diferentes artigos. Nesta tabela foram criadas três categorias de características de vizinhança: (1) segurança em relação a crimes; (2) segurança em relação a tráfego; (3) esté-

TABELA 4 - Artigos selecionados para revisão sistemática que foram classificados segundo agrupamento de medidas de característica de vizinhança, tipos de atividades físicas como desfecho e medidas de associação respectiva de cada estudo com IC95\%.

\begin{tabular}{|c|c|c|c|c|c|c|c|}
\hline \multirow[b]{2}{*}{ Artigo } & \multirow[b]{2}{*}{$\begin{array}{c}\text { Medida } \\
\text { de } \\
\text { Associação }\end{array}$} & \multicolumn{2}{|c|}{ Segurança em relação a crimes } & \multicolumn{2}{|c|}{ Estética e infra-estrutura } & \multicolumn{2}{|c|}{ Segurança em relação a tráfego } \\
\hline & & $\begin{array}{c}\text { Atividade física } \\
\text { global / ou de } \\
\text { lazer }\end{array}$ & $\begin{array}{l}\text { Caminhada / } \\
\text { Atividade física } \\
\text { de transporte }\end{array}$ & $\begin{array}{l}\text { Atividade física } \\
\text { global / ou de } \\
\text { lazer }\end{array}$ & $\begin{array}{l}\text { Caminhada / } \\
\text { Atividade física } \\
\text { de transporte }\end{array}$ & $\begin{array}{c}\text { Atividade física } \\
\text { global / ou de } \\
\text { lazer }\end{array}$ & $\begin{array}{l}\text { Caminhada / } \\
\text { Atividade física } \\
\text { de transporte }\end{array}$ \\
\hline $\begin{array}{l}\text { Amorim et al. } \\
(2010)\end{array}$ & $\mathrm{RP}$ & $1.10(1.0-1.2)$ & $1.10(1.00-1.22)$ & $\begin{array}{l}0.95(0.87-1.04) \\
1.01(0.92-1.10)\end{array}$ & $0.86(0.75-0.97)$ & $0.98(0.90-1.08)$ & $1.18(1.04-1.33)$ \\
\hline $\begin{array}{l}\text { Beenackers et } \\
\text { al. (2011) }\end{array}$ & OR & $0.57(0.4-0.7)$ & - & - & - & - & - \\
\hline $\begin{array}{l}\text { Bergman et al. } \\
\text { (2009) }\end{array}$ & OR & $0.77(0.52-1.14)$ & $1.64(1.14-2.36)$ & $0.77(0.52-1.14)$ & $0.67(0.47-0.95)$ & $1.02(0.68-1.51)$ & $0.85(0.59-1.22)$ \\
\hline $\begin{array}{l}\text { Corseuil et al. } \\
\text { (2012) }\end{array}$ & $\mathrm{RP}$ & $1.25(1.01-1.54)$ & $1.89(1.28-2.80)$ & - & - & - & - \\
\hline $\begin{array}{l}\text { Giehl et al. } \\
\text { (2012) }\end{array}$ & $\mathrm{RP}$ & $1.11(0.94-1.31)$ & - & $\begin{array}{l}1.25(1.03-1.43) \\
1,22(0,97 ; 1,53) \\
0,81(0,61 ; 1,07) \\
0,99(0,80 ; 1,23)\end{array}$ & - & $0,98(0,82 ; 1,18)$ & - \\
\hline $\begin{array}{l}\text { Hoehner et al. } \\
\text { (2005) }\end{array}$ & OR & $1.10(0.70-1.7)$ & $1.0(0.5-1.9)$ & $1.4(0.7-2.4)$ & $0.8(0.4-1.7)$ & $0.9(0.5-1.6)$ & $0.6(0.3-1.2)$ \\
\hline $\begin{array}{l}\text { Inoue et al. } \\
\text { (2009) }\end{array}$ & OR & $1.37(0.92-2.04)$ & $1.30(0,87-1,94)$ & $1.57(1.04-2.36)$ & $1.65(1.13-2.42)$ & $1.01(0.69,1.48)$ & $0.80(0.55,1.17)$ \\
\hline $\begin{array}{l}\text { Kamada et al. } \\
\text { (2009) }\end{array}$ & OR & $1.10(0.70-1.72)$ & - & $\begin{array}{l}2.05(1.30-3.22) \\
1.69(1.09-2.63)\end{array}$ & - & $0.93(0.59-1.47)$ & - \\
\hline $\begin{array}{l}\text { Oyeyemi et al. } \\
\text { (2012) }\end{array}$ & OR & $\begin{array}{l}0.34(0.06-0.91) £ \\
1.68(1.07-3.64) \#\end{array}$ & $\begin{array}{l}5.92(1.38-60.5) £ \\
7.00(2.71-18.0) \#\end{array}$ & - & - & $2.28(1.13-6.25)$ & $1.19(0.19-7.12)$ \\
\hline $\begin{array}{l}\text { Parra et al. } \\
\text { (2011) }\end{array}$ & OR & $1.30(1.00-1.70)$ & $\begin{array}{c}0.9(0.60-1.30) * * \\
1.50(1.00-2.10) \# \#\end{array}$ & $1.40(1.00-1.80)$ & $\begin{array}{l}1.2(0.9-1.7)^{* *} \\
1.0(0.8-1.4) \# \#\end{array}$ & $1.10(0.80-1.70)$ & $\begin{array}{c}0.6(0.4-0.9)^{* *} \\
1.3(0.9-1.9) \# \#\end{array}$ \\
\hline $\begin{array}{l}\text { Rech et al. } \\
\text { (2012) }\end{array}$ & $\mathrm{RP}$ & $1.05(0.98-1.13)$ & $\begin{array}{l}0.99(0.94-1.06)^{* *} \\
1.11(0.77-1.60) \# \#\end{array}$ & - & - & - & - \\
\hline $\begin{array}{l}\text { Sallis et al. } \\
\text { (2009) }\end{array}$ & OR & $\begin{array}{c}\text { Não significante } \\
\text { (OR não } \\
\text { mostrada) }\end{array}$ & - & $\begin{array}{l}1.47(1.32-1.65) \\
1.21(1.10-1.33)\end{array}$ & - & $1.32(1.16-1.54)$ & - \\
\hline $\begin{array}{l}\text { Santos et al. } \\
\text { (2008) }\end{array}$ & OR & $\begin{array}{c}1.01(0.86-1.20)+ \\
1.0(0.83-1.19) *\end{array}$ & - & $\begin{array}{l}1.31(1.12-1.55)_{+} \\
1.04(0.87-1.23)^{*}\end{array}$ & - & - & - \\
\hline $\begin{array}{l}\text { Tsunoda et al. } \\
\text { (2012) }\end{array}$ & OR & $0.99(0.61-1.62)$ & $0.94(0.60-1.48)$ & $\begin{array}{l}1.76(1.07-2.89) \\
1.67(1.07-2.62) \\
2.13(1.35-3.35)\end{array}$ & $2.00(1.33-3.02)$ & $1.29(0.81-2.05)$ & $1.46(0.96-2.21)$ \\
\hline $\begin{array}{l}\text { Velasquez et al. } \\
\text { (2009) }\end{array}$ & OR & $\begin{array}{l}2.17(1.11-4.21)_{+} \\
1.23(0.53-2.87)^{*}\end{array}$ & - & $\begin{array}{c}3,79(1,93-7,45)+ \\
1,27(0.47-3,44) *\end{array}$ & - & - & - \\
\hline
\end{tabular}

*Homens; +Mulheres; £ Diurno ; \# Noturno ; ${ }^{* *}$ Caminhada; \#\# Atividade física de transporte; - categoria não analisada 
tica e infra-estrutura. As medidas de associações dos estudos foram distribuídas conforme as características de vizinhança que mais se aproximavam dos constructos apresentados em uma das três categorias criadas. Quando o artigo tinha mais de um resultado que se encaixava na mesma categoria todos foram incluídos na tabela. As medidas de atividade física foram distribuídas em duas categorias: (1) atividade física global/ ou atividade física de lazer (nenhum estudo avaliou simultaneamente as duas categorias); e (2) caminhada / atividade física de transporte (apenas dois estudos analisaram este desfecho em separado e estes resultados foram incluídos na tabela).

\section{DISCUSSÃO}

A análise da associação entre prática de atividade física e características de vizinhança apontou diversas inconsistências entre os resultados dos estudos. Independentemente do instrumento utilizado para definir as características de vizinhança, ao analisar apenas o constructo de cada característica os resultados ainda são divergentes ou inconsistentes.

Um dos principais fatores de análise relativo a características de vizinhança, a "percepção de segurança em relação a crimes", é descrito na literatura como potencial determinante ou fator de influência na prática de caminhada tanto de lazer como de transporte e poderia também ser um determinante para a prática de atividade física em locais na vizinhança como parques e academias.

Todos os estudos incluídos na revisão analisaram este constructo (sendo em quatro artigos a "percepção de segurança em relação a crimes”, foco específico do estudo) porém apenas dois dos oito artigos que utilizaram IPAQ-E, seis dos oito artigos que utilizaram NEWS e um dos três artigos restantes, encontraram associação entre algum tipo de prática de atividade física e percepção de segurança em relação a crimes (total de $47.3 \%$ ).

Um segundo fator de característica de vizinhança importante e também analisado por todos os estudos incluídos na revisão é a "segurança em relação ao tráfego". Este fator estaria, em teoria, associado principalmente a caminhada (tanto de lazer como de transporte). Nos estudos que utilizaram IPAQ-E, há a construção de um constructo especifico de "segurança relativa a tráfego" e em apenas dois dos oito artigos analisados houve associação significativa entre este constructo e algum desfecho de prática de atividade física. Nos artigos que utilizaram NEWS a proposta de categorização original das perguntas para formação do constructo de "segurança relativa ao tráfego" foi utilizado em apenas quatro dos artigos e a associação significativa foi encontrada em apenas um deles. Os demais estudos apresentaram categorizações próprias ou apenas os resultados de alguma pergunta específica do questionário. Dos três artigos restantes, um analisou em conjunto a segurança relativa a crimes e ao tráfego, formando um escore único, e também não encontrou associação significativa. Dos outros dois artigos, um não encontrou associação e o outro não perguntou especificamente sobre trânsito, mas sobre questões relativas (como presença de calçadas e faixas de pedestre) e não encontrou associação significativa.

Assim, a diversidade de caracterizações entre os estudos para a formação de variáveis foi um dos principais problemas encontrados para a análise da associação entre atividade física e características de vizinhança. Alguns estudos justificam a não utilização das categorias originais (propostas pelos autores 
dos instrumentos) por estas não apresentarem boa consistência interna nas populações específicas analisadas, utilizando assim de PCAs (ou outras análises) para formação de novas categorias ou exclusão de alguma pergunta do questionário original. Alguns estudos que tinham foco específico de análise (dentro das características de vizinhança, como por exemplo segurança) utilizaram perguntas selecionadas do questionário e apresentaram os resultados por estas perguntas individualmente ou por categorizações próprias propostas pelos autores de cada artigo. Porém, nenhum dos estudos citados especifica se as categorizações foram propostas a priori (por um modelo teórico) ou se foram criadas durante as análises, buscando categorizações que encontrassem uma associação significante.

"Atributos estéticos da vizinhança" e "infra-estrutura da vizinhança" também são foco de interesse para associação com prática de atividade física, porém estas duas categorias se sobrepõe ao longo dos estudos. Algumas perguntas ou atributos da vizinhança como "presença de esgoto a céu aberto", "presença de lixo na rua", "presença de ciclovias", "iluminação pública", etc, são classificados indistintamente dentro destas duas categorias principalmente em estudos que utilizam NEWS como medida de característica de vizinhança.

Dos artigos que utilizaram IPAQ-E (instrumento com categorização original mais utilizada) apenas três artigos analisaram o atributo "estética da vizinhança" e em dois deles foram encontradas associações significativas com prática de atividade física. Dos artigos que utilizaram NEWS, mesmo analisando de forma geral as categorizações criadas pelos autores ou as perguntas em separado (conforme apresentado nos artigos), não houve nenhuma associação significativa encontrada com a prática de atividade física, exceto com uma pergunta relativa à "existência de ciclovias, trilhas ou calçadas de fácil acesso" em um dos artigos.

"Presença de ciclovia" é tratada enquanto categoria pelo IPAQ-E (sendo analisado em seis artigos com associação significativa em cinco) assim como "presença de calçadas" (analisada em seis artigos com associação significativa em três).

Com relação à disponibilidade de locais para prática de atividade física na vizinhança, IPAQ-E trata este atributo como a categoria "acesso a locais para prática de atividade física" analisada em seis artigos e com associação encontrada em quatro. Para os artigos que utilizaram NEWS e os três demais, perguntas relativas à "presença de áreas verdes ou parques", "presença de quadras esportivas", "ocorrência de eventos esportivos na vizinhança" e "presença/ proximidade de centros de recreação" podem ser interpretados como locais para prática de atividade física. Em todos os 10 artigos restantes (que não utilizaram IPAQ-E) pelo menos umas destas perguntas (ou perguntas semelhantes) estiveram presentes, porém só foi encontrada associação significativa com algum desfecho de prática de atividade física em quatro destes estudos.

Os resultados dos estudos não indicam ainda uma sólida relação entre as características de vizinhança e a prática de atividade física. A diversidade de populações e de metodologias para mensuração de características de vizinhança podem ser um dos motivos das diferenças apontadas na análise das associações, porém não há elementos suficientes para confirmar esta hipótese, principalmente pela dificuldade em comparar os diversos estudos por conta das diferenças metodológicas para definição da variável de característica de vizinhança, citadas anteriormente 
Destaca-se que das cinco formas de se mensuração de características de vizinhança descritas na literatura ${ }^{31-34}$, três delas não poderiam fazer parte da revisão (utilização de dados de censo, observação sistemática por avaliadores da vizinhança, medidas de disponibilidade e acessibilidade através de indicadores geográficos) devido ao critério de inclusão de artigos que exigia a aplicação de um questionário ou escala validada aos participantes do estudos.

Com relação às duas formas restantes de mensuração de características de vizinhança, não foram encontrados estudos que utilizassem informações obtidas através de questionários em uma perspectiva de percepção agregada de contexto de vizinhança, ou seja, uma análise georeferenciada das perspectivas dos moradores de uma mesma região. Todos os estudos encontrados tratavamse de estudos seccionais que utilizaram modelos de regressão logística (em alguns casos, multinomiais), modelos de regressão de poisson, um estudo que utilizou modelo aditivo generalizado misto e apenas um estudo que utilizou análise multinível, porém neste apenas variáveis fornecidas por estatísticas oficiais eram georeferenciadas e não as percepções dos participantes do estudo.

Este resultado aponta uma lacuna importante nos estudos de associação entre a prática de atividade física e características de vizinhança. Quando há níveis de análise (como as vizinhanças) em grupos de indivíduos, o uso de modelos de regressão tradicionais (que assumem independência das observações) pode levar à subestimação de erros padrões e a associações imprecisas, recomendando-se o uso de modelos multiníveis ${ }^{35}$. Assim, indivíduos pertencentes a uma mesma vizinhança, supostamente, deveriam ter a percepção das características de sua vizinhança semelhantes, o que diminui a variabilidade entre eles, pois estes compartilham às mesmas características do ambiente físico e social.

O não uso de análises multiníveis nos estudos analisados pode ter contribuído para algumas das associações não significativas ou para as inconsistências encontradas entre os estudos. Assim, recomenda-se que estudos futuros levem em consideração esta abordagem ao analisar a associação entre características de vizinhança e a prática de atividade física.

Apesar das diversas inconsistências encontradas nas análises estatísticas, comparando os diferentes estudos, destaca-se que a maior parte das associações encontradas foi no sentido esperado (ou seja, quanto melhor a percepção em relação à vizinhança maior a prática de atividade física). Este é um resultado importante que demonstra a necessidade de melhoria das condições de vizinhanças, em diversos aspectos, tendo em vista a possível relação direta entre as variáveis de características de vizinhança e atividade física. Programas governamentais que busquem melhorar as condições coletivas (como redução da criminalidade, adequado controle de tráfego, melhoria na iluminação pública, melhoria na mobilidade, etc.) podem ter um efeito direto, mesmo que não seja esse o objetivo inicial, no aumento da prática de atividade física, e consequentemente na redução da prevalência de inativos, trazendo assim ganhos em relação ao estado de saúde da população em geral.

\section{REFERÊNCIAS}

1. Pitsavos C, Panagiotakos DB, Lentzas Y, Stefanadis C. Epidemiology of leisure-time physical activity in socio-demographic, lifestyle and psychological characteristics of men and women in Greece: the ATTICA Study.BMC Public Health. 2005; 5(1): 37-45. 
2. Guedes DP, Goncalves LAV. Impacto da prática habitual de atividade física no perfil lipídico de adultos. Arq Bras Endocrinol Metab. 2007; 51(1): 72-78.

3. COSTA, MAP; VASCONCELOS, AGG, FONSECA, MJM. Prevalência de obesidade, excesso de peso e obesidade abdominal e associação com prática de atividade física em uma universidade federal. Rev. bras. epidemiol. 2014; 17 (2): 421-436.

4. Pan SY, Cameron C, Desmeules M, Morrison H, Craig CL, Jiang X. Individual, social, environmental, and physical environmental correlates with physical activity among Canadians: a cross-sectional study. BMC Public Health.2009; 9(1): 21-33.

5. Neutzling MB, Rombaldi AJ, Azevedo MR, Hallal PC. Fatores associados ao consumo de frutas, legumes e verduras em adultos de uma cidade no Sul do Brasil.Cad. Saúde Pública. 2009; 25 (11): 2365-74.

6. Bauman A, Bull F, Chey T, Craig CL, Ainsworth BE, Sallis JF, et al. The International Prevalence Study on Physical Activity: results from 20 countries. Int J Behav Nutr Phys Act. 2009; 6(1): 21-32.

7. Pickett KE, Pearl M. Multilevel analyses of neighbourhood socioeconomic context and health outcomes: a critical review. J Epidemiol Community Health. 2001; 55 (1): 111-22.

8. Caiaffa WT, Ferreira FR, Ferreira AD, Oliveira CDL, Camargos VP, Proietti FA. Saúde urbana: a cidade é uma estranha senhora, que hoje sorri e amanhã te devora. Cien Saude Colet. 2008; 13(6):1785-96.

9. Cummins S, Curtis S, Diez-Roux AV, Macintyre S. Understanding and representing place' in health research: A relational approach. Soc Sci Med. 2007; 65(1):1825-38.

10. Corseuil MW, Hallal PC, Corseuil HX, Schneider IJC, d'Orsi E. Safety from Crime and Physical Activity among Older Adults: A Population-Based Study in Brazil. J Environ Public Health. 2012; 2012:641010.

11. 11- Lee RE, Mama SK, Medina AV, Ho A, Adamus HJ. Neighborhood factors influence physical activity among community dwelling African American and Hispanic or Latina women. Health \& Place. 2012; 18 (1): 63-70.

12. Higgins JP, Green S. Cochrane Handbook for Systematic Reviews of Interventions [Internet]. Version 5.1.0. The Cochrane Collaboration; 2011. Disponível em: www. cochrane-handbook.org.

13. Kamada M, Kitayuguchi J, Inoue S, Kamioka H, Mutoh Y, Shiwaku K. Environmental correlates of physical activity in driving and non-driving rural Japanese women. Prev Med. 2009; 49(6): 490-6.

14. Tsunoda K, Tsuji T, Kitano N, Mitsuishi Y, Yoon JY, YOON J, OKURA T. Associations of physical activity with neighborhood environments and transportation modes in older Japanese adults. Prev Med. 2012; 55(2): 113-8.

15. Inoue S, Murase N, Shimomitsu T, Ohya Y, Odagiri Y, Takamiya T,et al. Association of physical activity and neighborhood environment among Japanese adults. Prev Med. 2009; 48(4): 321-5.

16. Oyeyemi AL, Adegoke BO, Sallis JF, Oyeyemi AY, Bourdeaudhuij ID. Perceived crime and traffic safety is related to physical activity among adults in Nigeria. Bmc Public Health. 2012; 12 (1): 94-105.

17. Wallmann B, Bucksch J, Froboese I. The association between physical activity and perceived environment in German adults. European Journal of Public Health.2012; 22(4): 502-8.

18. Santos R, Silva P, Santos P, Ribeiro JC, Mota J. Physical activity and perceived environmental attributes in a sample of Portuguese adults: Results from the Azorean Physical Activity and Health Study. Prev Med. 2008; 47(1): 83-88.

19. Bergman P, Grjibovski AM, Hagstromer M, Sallis JF, Sjostrom M. The association between health enhancing physical activity and neighbourhood environment among Swedish adults. a population-based cross-sectional study. Int J Behav Nutr Phys Act. 2009; 6(1): 8-15.

20. Sallis J, Bowles H, Bauman A, Ainsworth, B., Bull FC, Craig CL, et al. Neighborhood Environments and Physical Activity Among Adults in 11 Countries. Am J Prev Med. 2009; 36(6): 484-90. 
21. Rech CR, Reis RS, Hino AAF, Rodriguez-Añez CR, Fermino RC, Gonçalves PB,et al. Neighborhood safety and physical inactivity in adults from Curitiba, Brazil. Int J Behav Nutr Phys Act. 2012; 9(1): 72-80.

22. Parra DC, Hoehner CM, Hallal PC, Ribeiro IC, Reis R, Brownson RC, et al. Perceived environmental correlates of physical activity for leisure and transportation in Curitiba, Brazil. Prev Med. 2011; 52(3): 234-8.

23. Amorim TC, Azevedo MR, Hallal PC. Physical Activity Levels According to Physical and Social Environmental Factors in a Sample of Adults Living in South Brazil. J Phys Act Health. 2010; 7 (1): 204-12.

24. Florindo AA., Salvador EP, Reis RS, Guimaraes VV. Perception of the environment and practice of physical activity by adults in a low socioeconomic area. Rev Saude Publica. 2011; 45(2): 302-10.

25. Salvador EP, Florindo AA, Reis RS, Costa EF. Perception of the environment and leisure-time physical activity in the elderly. Rev Saude Publica. 2009; 43(6): 972-80.

26. Giehl MWC, Schneider IJC, Corseuil HX, Benedetti TRB, d'Orsi E. Physical activity and environment perception among older adults: a population study in Florianopolis, Brazil. Rev Saude Publica. 2012; 46(3): 516-25.

27. Van Dyck D, Cerin E, Conway T, Bourdeaudhuij ID, Owen N, Kerr J, et al. Perceived neighborhood environmental attributes associated with adults' leisure-time physical activity: Findings from Belgium, Australia and the USA. Health and Place. 2013; 19(1): 59-68.

28. Velasquez K, Holahan C, You X. Relationship of perceived environmental characteristics to leisure-time physical activity and meeting recommendations for physical activity in Texas. Prev Chronic Dis. 2009; 6(1): A24.

29. Beenackers MA, Kamphuis CBM, Burdorf A, Mackenbach JP,LENTHE FJ. Sports participation, perceived neighborhood safety, and individual cognitions: how do they interact? Int J Behav Nutr Phys Act. 2011; 8 (1): 76-84.

30. Hoehner CM, Ramirez LKB, Elliott MB, Handy SL, Brownson RC. Perceived and objective environmental measures and physical activity among urban adults. Am J Prev Med. 2005; 28(2): 105-16.

31. Sampson RJ, Raudenbush SW. Systematic social observation of public spaces: a new look at disorder in urban neighborhoods. Am J Sociol. 1999; 105(3): 603-51.

32. Giles-Corti B, Donovan RJ. Socioeconomic status differences in recreational physical activity levels and real and perceived Access to a supportive physical environment. Prev Med. 2002; 35 (6): 601-11.

33. Pikora TJ, Bull FC, Jamrozik K, Knuiman M, Giles-Corti B, Donovan RJ. Developing a reliable audit instrument to measure the physical environment for physical activity. Am J Prev Med. 2002; 23 (3): 187-94.

34. Echeverria SE, Diez-Roux AV, Link BG. Reliability of self-reported neighborhood characteristics. J Urban Health. 2004; 81(4): 682-701.

35. Rasbash J, Steele F, Browne WJ, Goldstein H. A user>s guide to MLwiN: version 2.0. London: Center for Multilevel Modeling, Institute of Education, 2009.

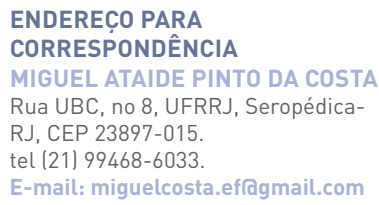

